\title{
30 Jahre Deutsche Einheit - welche Lehren zieht die Wirtschaftsforschung?
}

\begin{abstract}
Der Tag der Deutschen Einheit jährte sich 2020 zum 30. Mal. Der Weg der Vereinigung ist nicht immer geradlinig verlaufen. Auf der gemeinsamen Konferenz von Wirtschaftsdienst/ZBW und dem Leibniz-Institut für Wirtschaftsforschung Halle (IWH) am 4. November 2020 wurden die Entwicklungen nach der Wiedervereinigung in einem zeithistorischen Kontext betrachtet und die aktuellen Herausforderungen identifiziert, vor denen der gesamtdeutsche Prozess der Zusammenführung heute steht. Neben den unbestreitbaren Erfolgen der ersten Jahre standen Themen wie Produktivitätsunterschiede, Probleme bei der Privatisierung des Treuhandvermögens und eine gegebenenfalls fehlende Industriepolitik für Ostdeutschland, die den Konvergenzprozess erschwert haben könnte, im Fokus. Das vorliegende Konferenzheft des Wirtschaftsdienst ist auf Grundlage dieser gemeinsamen Konferenz mit dem IWH entstanden. Das IWH war mit seiner Expertise in Fragen der Wiedervereinigung und des anschließenden Transformationsprozesses genau die richtige Partnerinstitution für die jährliche Konferenz des Wirtschaftsdienst.
\end{abstract}

Nach einer Eröffnung durch Christian Breuer und Oliver Holtemöller folgte eine Keynote von Christoph Bergner über die politischen Erfahrungen bei der wirtschaftlichen und gesellschaftlichen Transformation im Zuge des deutschen Vereinigungsprozesses. Als ehemaliger Ministerpräsident von Sachsen-Anhalt und ehemaliger Beauftragter der Bundesregierung für die neuen Bundesländer verfügt Christoph Bergner über vielfache, auch persönliche Erfahrungen im Zusammenhang mit der Transformation Ostdeutschlands.

(C) Der/die Autor:in(nen) 2021. Open Access: Dieser Artikel wird unter der Creative Commons Namensnennung 4.0 International Lizenz veröffentlicht (creativecommons.org/licenses/by/4.0/deed.de).

Open Access wird durch die ZBW - Leibniz-Informationszentrum Wirtschaft gefördert.

Dr. Christian Breuer ist Chefredakteur von Wirtschaftsdienst und Intereconomics in der ZBW Leibniz-Informationszentrum Wirtschaft in Hamburg.

Cora Wacker-Theodorakopoulos ist Redakteurin beim Wirtschaftsdienst in der ZBW.
Dalia Marin von der TU München betrachtet den historischen Konvergenzprozess. Sie arbeitet die Unterschiede zwischen West- und Ostdeutschland sowie Probleme im Wiedervereinigungsprozess heraus und sieht optimistische Zukunftsperspektiven. Einen vertieften Einblick in die Geschichte der viel diskutierten Treuhandanstalt bietet Marcus Böick von der Ruhr-Universität Bochum. Er sieht die neue Transformationsforschung als interdisziplinäre Aufgabe. Rüdiger Pohl, ehemaliger Präsident des IWH und Mitglied im Sachverständigenrat von 1986 bis 1994, beschreibt in seinem Beitrag die Geschichte der Wirtschafts- und Währungsunion sowie die Herausforderungen bei der Transformation und dem Wiederaufbau der ostdeutschen Wirtschaft.

Steffen Müller (IWH) analysiert den Konvergenzprozess zwischen Ost- und Westdeutschland aus einer mikroökonomischen Perspektive. Eva Dettmann (IWH) und ihre Koautoren diskutieren die Zuschüsse der Gemeinschaftsaufgabe Regionale Wirtschaftsstruktur (GRW). Sie kommen in ihrer Analyse zu dem Ergebnis, dass die Investitionszuschüsse zur wirtschaftlichen Entwicklung in Ostdeutschland beigetragen haben. Lukas Mergele (ifo Institut) und seine Koautoren untersuchen die Unterschiede zwischen Ost- und Westdeutschland aus einer historischen Perspektive. Sie können zeigen, dass sich sozioökonomische Faktoren bereits vor der deutschen Teilung zwischen West- und Ostdeutschland unterschieden haben.

Anstatt der sonst bei Konferenzen des Wirtschaftsdienst abschließenden Podiumsdiskussion, gab es dieses Mal eine Online-Diskussionsrunde „Aufbau Ost: Fehler der Vergangenheit und Chance in der Zukunft", die zu einem lebhaften Austausch über einzelne Aspekte des Wiedervereinigungsprozesses führte. Dabei wurden unter anderem die Schwierigkeiten der Messung realer Konvergenz erörtert. Zudem wurden die Erfolge der industriepolitischen Strategie der 1990er Jahre sowie Perspektiven bei der Energie- und Verkehrswende diskutiert, die als Chance für die ostdeutsche Wirtschaft des 21. Jahrhunderts gesehen werden können.

Den Schlusspunkt in diesem Konferenzheft setzt Oliver Holtemöller und zeigt, dass das BIP pro Kopf kein geeigneter Indikator für den Konvergenzprozess darstellt. Er schlägt vor, auf andere Merkmale wie z. B. Arbeitsproduktivität und auf Wohlfahrtsindikatoren als Maß für die Angleichung der Lebensverhältnisse abzustellen. 